\title{
Robotic adrenalectomy versus laparoscopic adrenalectomy for pheochromocytoma: a systematic review and meta-analysis
}

\author{
Lei Du, Zhan Yang, Jinchun Qi, Yaxuan Wang \\ Department of Urology, The Second Hospital of Hebei Medical University, Shijiazhuang, China
}

\author{
Videosurgery Miniinv 2022; 17 (1): 1-8 \\ DOI: https://doi.org/10.5114/wiitm.2021.107764
}

\begin{abstract}
Introduction: The application of robotic adrenalectomy $(R A)$ has been increasing. However, there is still controversy about whether RA is more feasible than laparoscopic adrenalectomy (LA) for pheochromocytoma (PHEO).

Aim: To evaluate the efficacy and safety of RA vs. LA for PHEO.

Material and methods: A literature search of the PubMed, Ovid, and Scopus databases was performed to identify eligible studies up to April 2021. All studies comparing RA versus LA for PHEO were included. Data were analysed using the Cochrane Collaboration's Review Manager (RevMan) 5.4 software.

Results: Overall, 4 studies including 386 patients (RA 155; LA 231) were included. RA might have larger tumour size $(W M D=0.72 \mathrm{~cm}, 95 \% \mathrm{Cl}: 0.31$ to $1.13 ; p<0.001)$. There were no statistically significant differences in operative time (WMD $=-12.49 \mathrm{~min}, 95 \% \mathrm{Cl}:-29.50$ to $4.52 ; p=0.15)$, estimated blood loss (EBL) $(W M D=-28.48 \mathrm{ml}, 95 \% \mathrm{Cl}$ : $-58.92,1.95 ; p=0.07)$, transfusion rate $(O R=0.70,95 \% \mathrm{Cl}: 0.07$ to $7.07 ; p=0.77)$, or conversion rate $(O R=0.44$, $95 \%$ Cl: 0.07 to $2.88 ; p=0.39)$. There were no significant differences between the 2 groups in terms of postoperative complications (OR $=1.06,95 \% \mathrm{Cl}$ : 0.62 to $1.82 ; p=0.84$ ) and Clavien Dindo score $\geq 3$ complications $(O R=1.15$, 95\% Cl: 0.39 to 3.41; $p=0.80$ ). Patients from the RA group could benefit from shorter length of hospital stay $(W M D=-0.51$ days, $95 \% \mathrm{Cl}-0.91$ to $-0.12 ; p=0.01)$.
\end{abstract}

Conclusions: RA is a feasible, safe, and comparable treatment option for PHEO.

Key words: robotic adrenalectomy, laparoscopic adrenalectomy, pheochromocytoma, meta-analysis.

\section{Introduction}

Pheochromocytoma (PHEO) is a rare neuroendocrine tumour that originates in the adrenal medulla or extra-adrenal glands [1]. In the general population, the estimated incidence of PHEO is between $0.05 \%$ and $0.1 \%$ [2]. Typical clinical manifestations include paroxysmal hypertension, sweating, headache, and palpitations caused by excessive catecholamine secretion, which endanger patients' lives [3]. Adrenalectomy is considered the gold standard for PHEO [1, 4]. Open adrenalectomy has been considered the gold standard for a long time. However, laparoscopic ad- renalectomy (LA) has replaced open adrenalectomy as the "gold standard" since the 1990s [5]. With the widespread application of robotic surgery, the application of robotic adrenalectomy (RA) is increasing. Robotic surgery has the following advantages: wristed instrument to eliminate tremors, flexible and intuitive instrument control, and highly visual three-dimensional vision [6]. However, the longer learning curve, longer operative time, and higher costs limit the application of robotic surgery [7]. In addition, due to the lack of tactile feedback during dissection of PHEO, RA could increase the risk of bleeding, cardiovascular accidents, and intraoperative hypertensive crisis [8].

\footnotetext{
Address for correspondence

Yaxuan Wang MD, Department of Urology, The Second Hospital of Hebei Medical University, Shijiazhuang, China,

e-mail: wangmnwk@126.com
} 
Whether RA is more feasible for PHEO than LA is still controversial. In order to better understand the ongoing debate, it is necessary to analyse the current evidence and draw objective conclusions. The goal of this study is to perform a meta-analysis to evaluate the efficacy and safety of RA versus LA when treating patients with PHEO.

\section{Aim}

The aim of the study is to evaluate the efficacy and safety of RA versus LA when treating patients with $\mathrm{PHEO}$ in terms of operative time, estimated blood loss $(E B L)$, transfusion, conversion rate, peri-operative haemodynamic outcomes, complications, Clavien-Dindo score $\geq 3$ complications, and length of hospital stay.

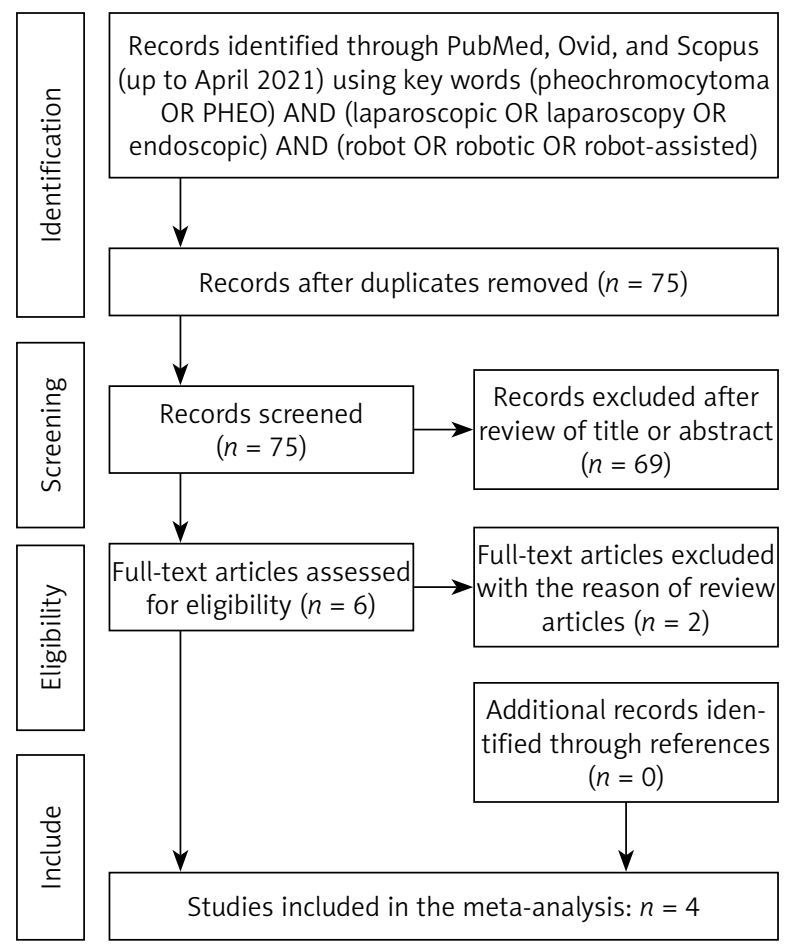

Figure 1. PRISMA flow diagram of the search strategy of the included studies

\section{Material and methods}

\section{Literature search}

A systematic literature search of the PubMed, Ovid, and Scopus databases (up to April 2021) was performed to identify studies comparing RA and LA. The search strategy was "(pheochromocytoma OR PHEO) AND (laparoscopic OR laparoscopy OR endoscopic) AND (robot OR robotic OR robot-assisted)". The Preferred Reporting Items for Systematic Reviews and Meta-analysis (PRISMA) criteria was used for article selection (Figure 1). Two investigators (LD and $Z Y$ ) screened the titles and abstracts of potentially eligible studies independently. Relevant references were also checked for additional studies.

\section{Inclusion and exclusion criteria}

Inclusion criteria: (1) original studies comparing RA to LA for PHEO; (2) written in English language; (3) adult studies only.

Exclusion criteria: (1) children's studies; (2) studies including paraganglioma; (3) studies without primary or sufficient data (i.e. reviews, commentaries, conference abstracts).

\section{Data extraction and quality assessment}

Data were extracted independently by 2 researchers (LD and ZY) using a predefined data extraction form, including demographic characteristics (age, gender, BMI, tumour size, and tumour side), peri-operative outcomes (operative time, EBL, transfusion, and conversion), peri-operative haemodynamic outcomes (greatest intraoperative systolic blood pressure, lowest intraoperative systolic blood pressure, and greatest heart rate), and post-operative outcomes (complications, Clavien-Dindo score $\geq 3$ complications, and length of hospital stay). Any discrepancy was resolved in consultation with JQ. Two researchers (LD and ZY) independently assessed

Table I. Basic characteristics of included studies in this meta-analysis

\begin{tabular}{|lllcccc|}
\hline Study & Period & Design & Region & \multicolumn{2}{c|}{ RA/LA } & LE \\
\cline { 1 - 1 } & & & & Cases $(n)$ & FU [month] & \\
\hline Aliyev 2013 & 2000-2012 & RTP & USA & $25 / 40$ & 12 & 4 \\
\hline Fang 2020 & $2000-2017$ & RTP & Multicentre & $41 / 89$ & NS & 4 \\
\hline Ma 2020 & $2016-2019$ & RCT & China & $70 / 70$ & NS & $2 b$ \\
\hline Fu 2020 & 2016-2019 & RTP & China & $19 / 32$ & $26.6 / 31.2$ & 4 \\
\hline
\end{tabular}

$R A$ - robotic adrenalectomy, LA - laparoscopic adrenalectomy, FU - follow-up, LE - level of evidence according to the Oxford Centre for Evidence-based Medicine, RTP - retrospective, RCT - randomized controlled trail, NS - not specified. 

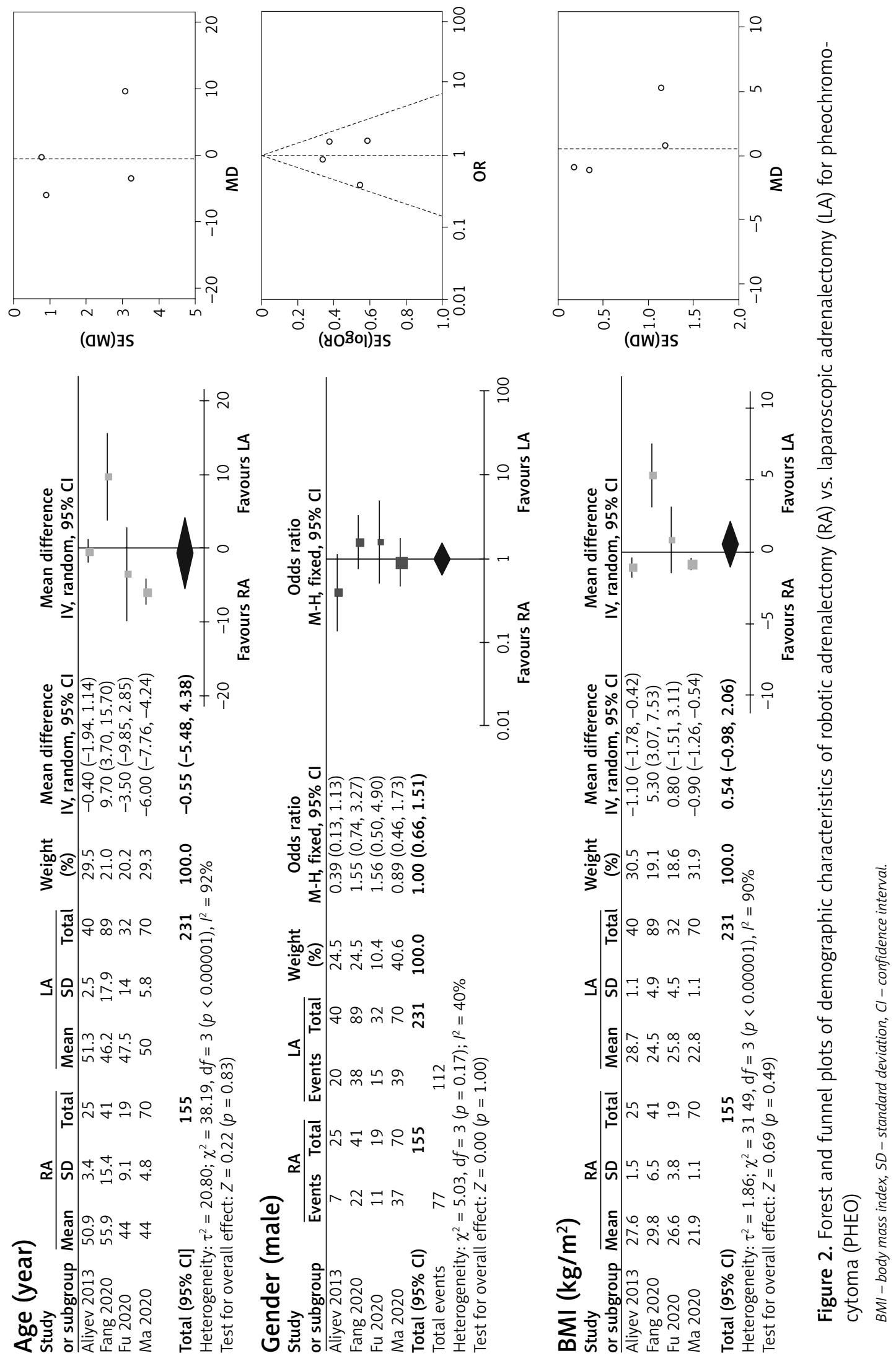

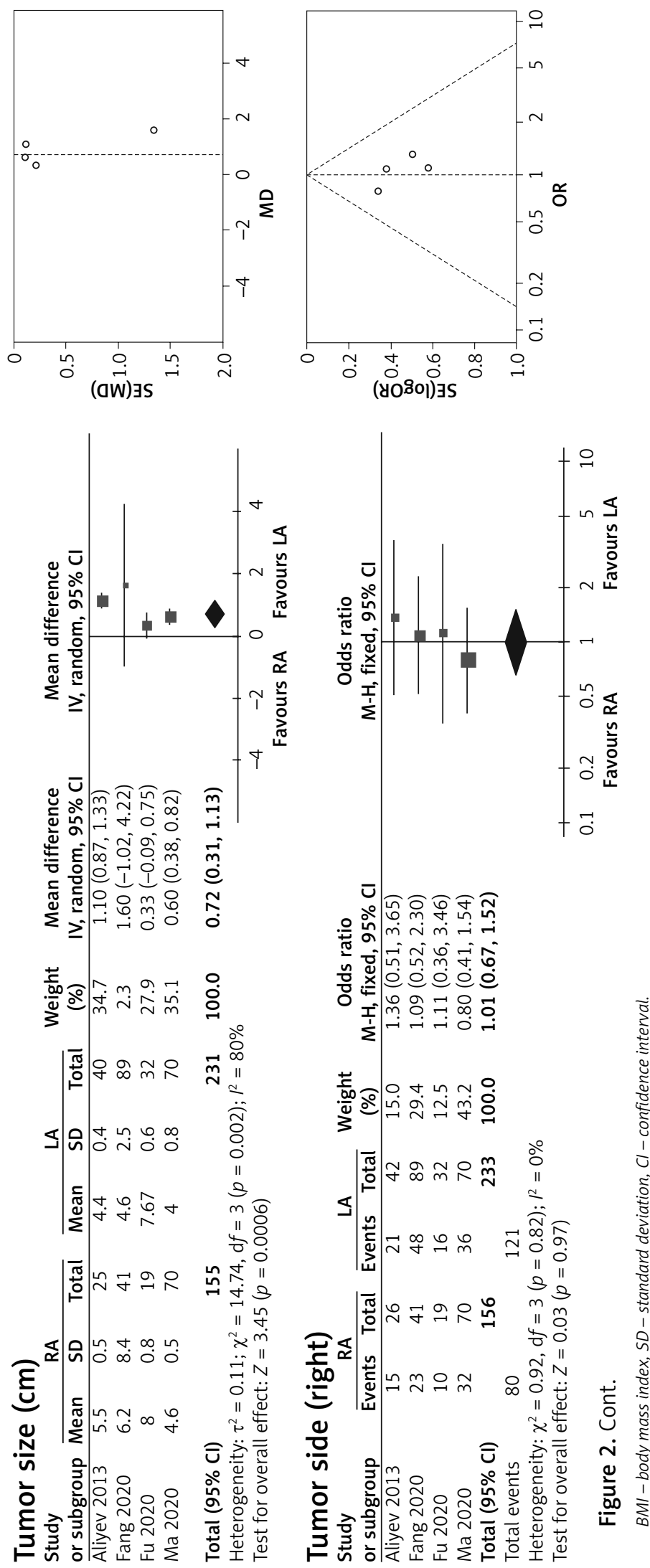

the evidence level of the included studies according to the Oxford Centre for Evidence-based Medicine (Table I).

\section{Statistical analysis}

Cochrane Collaboration's Review Manager (RevMan) 5.4 software (Cochrane Collaboration, Oxford, UK) was adopted to analyse the effects of the outcomes. Because only means and standard deviations are permitted for continuous data in RevMan 5.4 , a validated mathematical model was used to convert median (range) to mean (standard deviation) for studies only reporting medians and ranges [9]. Heterogeneity was defined based on the Cochrane $\mathrm{Q} p$-value and $l^{2}$ statistic. If $p>0.1$ or $l^{2}<50 \%$, a fixed-effects model was used. While, if $p<0.1$ or $p^{2}>50 \%$, a random-effects model was used. A $p<$ 0.05 was considered statistically significant. To evaluate publication bias, funnel plots were examined.

\section{Results}

Overall, 4 studies including 386 patients (RA 155; LA 231) were included in this study (Figure 1) [10-13]. Table I shows the basic characteristics and quality assessment of the included studies.

There were no statistically significant differences between RA and LA in age (WMD $=-0.55$ years, $95 \% \mathrm{Cl}:-5.48$ to $4.38 ; p=0.83)$, male component ( $\mathrm{OR}=1.00,95 \% \mathrm{Cl}: 0.66$ to $1.51 ; p=1.00), \mathrm{BMI}$ (WMD $=0.54 \mathrm{~kg} / \mathrm{m}^{2}, 95 \% \mathrm{Cl}:-0.98$ to $2.06 ; p=0.49$ ), and tumour side $(\mathrm{OR}=1.01,95 \% \mathrm{Cl}: 0.67$ to $1.52 ; p=$ 0.97) [10-13]. Meta-analysis of these studies showed larger tumour size in the RA group (WMD $=0.72 \mathrm{~cm}$, 95\% Cl: 0.31 to $1.13 ; p<0.001$ ) [10-13] (Figure 2).

All the 4 studies reported operative time and EBL [10-13]. There were no significant differences in operative time (WMD $=-12.49 \mathrm{~min}, 95 \% \mathrm{Cl}:-29.50$ to 4.52; $p=0.15)$ and EBL (WMD $=-28.48 \mathrm{ml}, 95 \% \mathrm{Cl}$ : $-58.92,1.95 ; p=0.07$ ) between the 2 groups. Two studies including 191 patients (RA 89; LA 102) reported transfusion $[12,13]$. The transfusion rate was similar between $\mathrm{RA}$ and $\mathrm{LA}(\mathrm{OR}=0.70,95 \% \mathrm{Cl}$ : 0.07 to $7.07 ; p=0.77)$. Two studies including 205 patients (RA 95; LA 110) reported open conversion $[10,13]$. A meta-analysis of these studies showed comparable conversion rates for RA and LA (OR = $0.44,95 \% \mathrm{Cl}: 0.07$ to $2.88 ; p=0.39$ ) (Figure 3 ).

Three studies including 338 patients (RA 137; LA 201) reported the greatest intraoperative systol- 
Operative time $(\mathrm{min})$

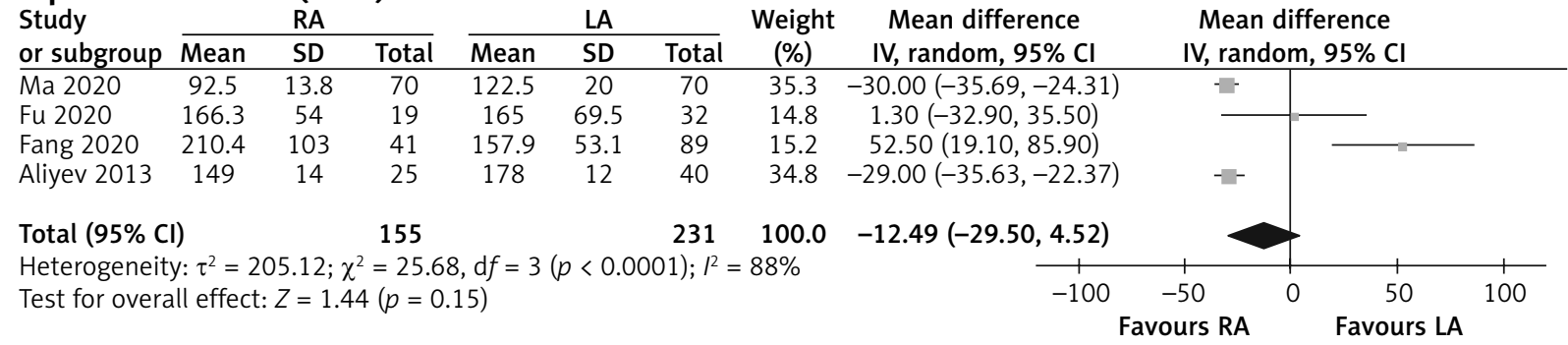

\begin{tabular}{|c|c|c|c|c|c|c|c|c|c|}
\hline EBL (ml) & & & & & & & & & \\
\hline Study & & RA & & & LA & & Weight & Mean difference & Mean difference \\
\hline or subgroup & $\overline{M e a n}$ & SD & Total & Mean & SD & Total & $(\%)$ & IV, random, $95 \% \mathrm{Cl}$ & $\mathrm{IV}$, random, $95 \% \mathrm{Cl}$ \\
\hline Aliyev 2013 & 36 & 12 & 25 & 43 & 10 & 40 & 34.2 & $-7.00(-12.63,-1.37)$ & $T$ \\
\hline Fang 2020 & 173 & 404 & 41 & 134 & 243 & 89 & 4.5 & $39.00(-94.57,172.57)$ & \\
\hline Fu 2020 & 100 & 37.5 & 19 & 200 & 55 & 32 & 27.9 & $-100.00(-125.45,-74.55)$ & $-\square-$ \\
\hline Ma 2020 & 100 & 15.6 & 70 & 100 & 37.5 & 70 & 33.5 & $0.00(-9.51,9.51)$ & \\
\hline Total $(95 \% \mathrm{Cl}$ & & & 155 & & & 231 & 100.0 & $-28.48(-58.92,1.95)$ & \\
\hline $\begin{array}{l}\text { Heterogeneity } \\
\text { Test for overa }\end{array}$ & $\begin{array}{l}y: \tau^{2}=\epsilon \\
\text { all effect }\end{array}$ & $\begin{array}{l}.18 \\
Z=1\end{array}$ & $\begin{array}{l}=53.5 \\
(p=0\end{array}$ & 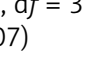 & & & & -2 & $\begin{array}{cc}-100 & 0 \\
\text { vours RA } & \text { Favours LA }\end{array}$ \\
\hline
\end{tabular}

\section{Transfusion}

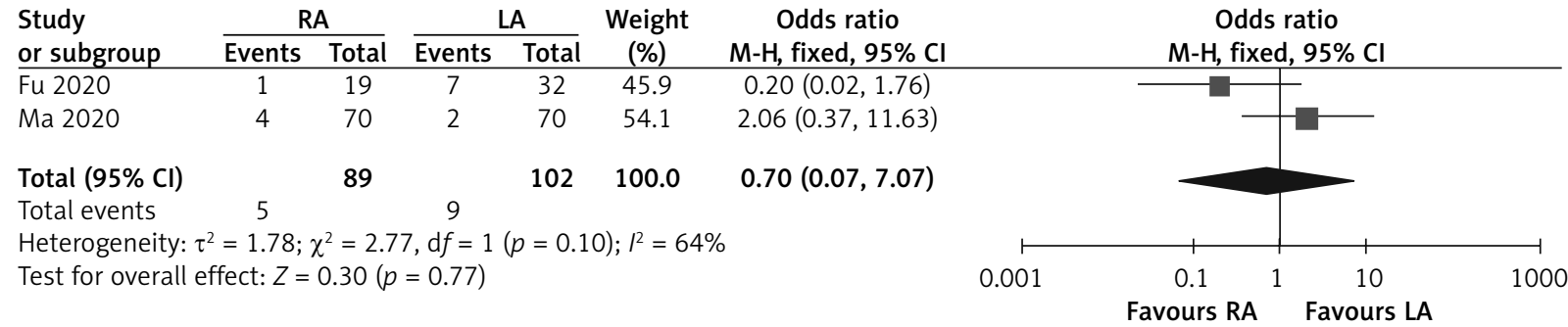

\section{Conversion}

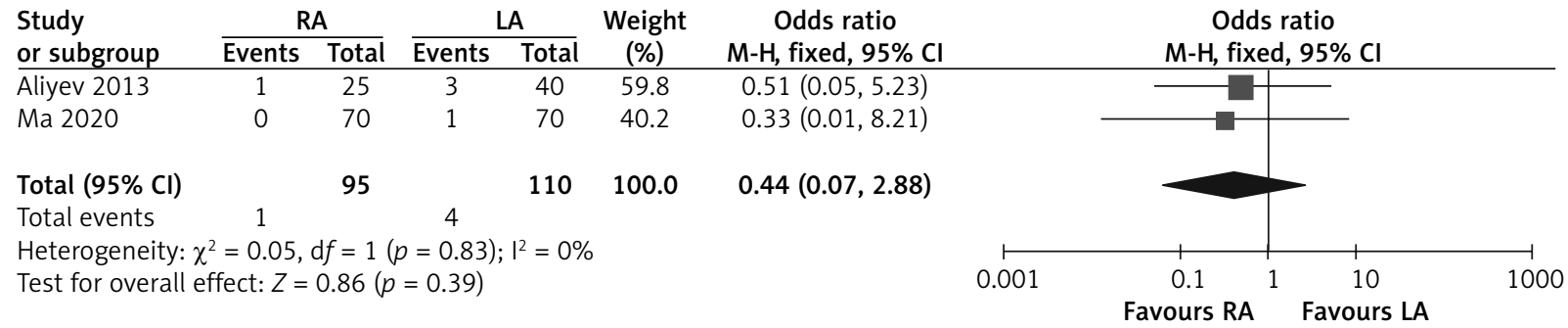

Figure 3. Forest and funnel plots of peri-operative outcomes of robotic adrenalectomy (RA) vs. laparoscopic adrenalectomy (LA) for pheochromocytoma (PHEO)

$E B L$ - estimated blood loss, SD - standard deviation, Cl - confidence interval.

ic blood pressure and lowest intraoperative systolic blood pressure [10, 11, 13]. A meta-analysis of these studies showed similar greatest intraoperative systolic blood pressure (WMD $=-0.05 \mathrm{~mm} \mathrm{Hg}, 95 \% \mathrm{Cl}$ : $-2.04,1.94 ; p=0.96)$ and lowest intraoperative systolic blood pressure (WMD $=-2.71 \mathrm{~mm} \mathrm{Hg}, 95 \% \mathrm{Cl}$ : -9.37, 3.95; $p=0.42$ ). Two studies including 208 patients (RA 96; LA 112) reported greatest heart rate $[10,13]$. There was no statistically significant differ- ence in greatest heart rate $(\mathrm{WMD}=0.00$ beat $/ \mathrm{min}$, 95\% Cl: $-1.17,1.17 ; p=1.00$ ) (Figure 4).

All the 4 studies reported postoperative complications, Clavien-Dindo score $\geq 3$ complications, and length of hospital stay [10-13]. There were no significant differences between the 2 groups in terms of postoperative complications (OR $=1.06,95 \% \mathrm{Cl}$ : 0.62 to $1.82 ; p=0.84)$ and Clavien-Dindo score $\geq 3$ complications ( $\mathrm{OR}=1.15,95 \% \mathrm{Cl}$ : 0.39 to 3.41 ; 


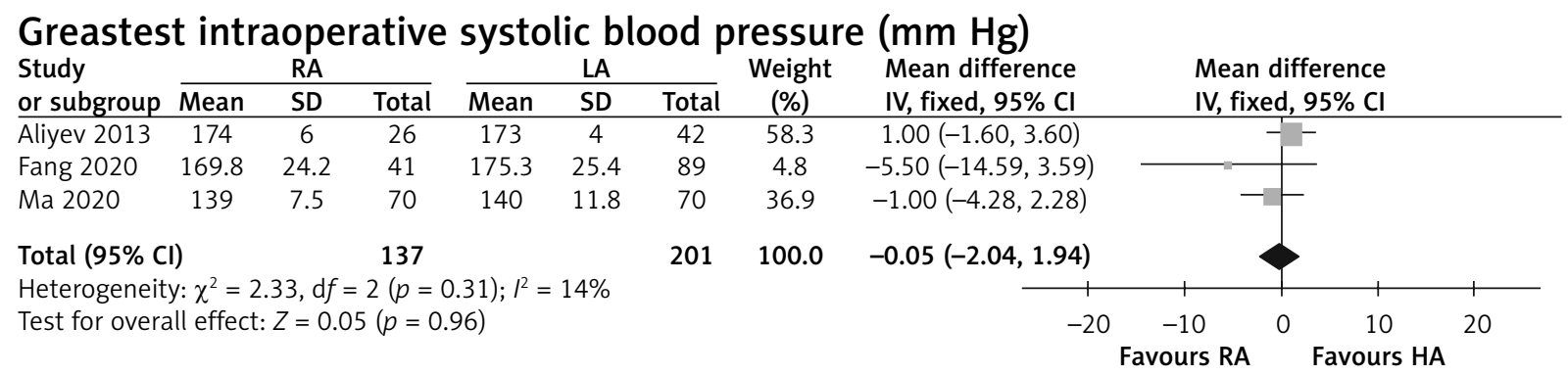

Lowest intraoperative systolic blood pressure $(\mathrm{mm} \mathrm{Hg})$

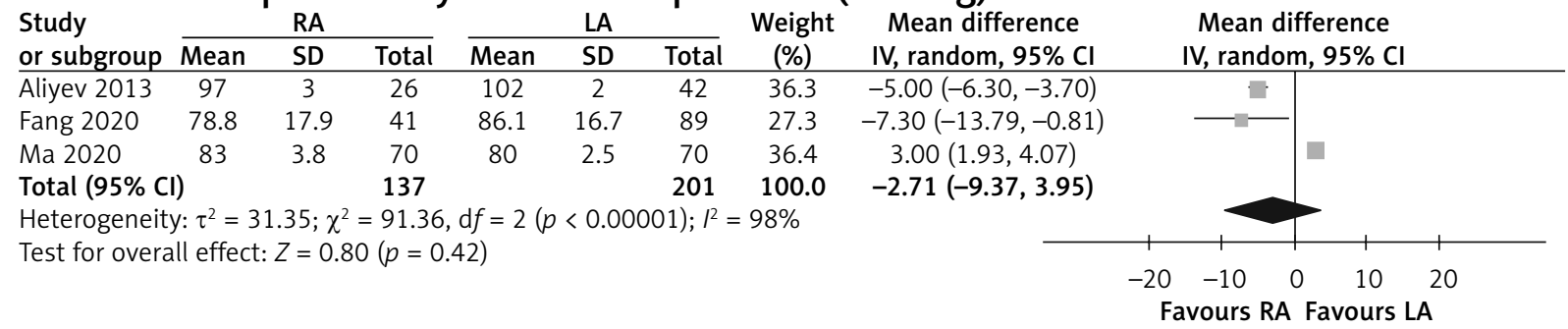

\section{Greatest heart rate (beat/min)}

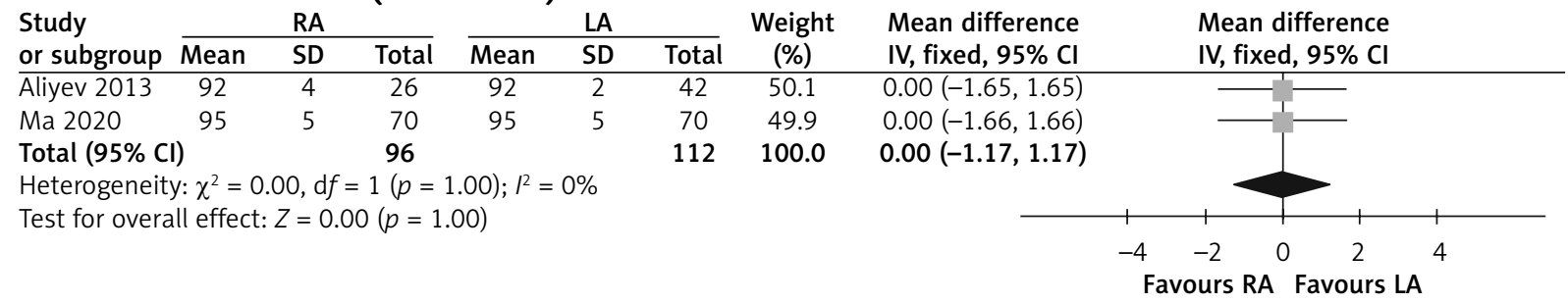

Figure 4. Forest and funnel plots of peri-operative hemodynamic outcomes of robotic adrenalectomy (RA) vs. laparoscopic adrenalectomy (LA) for pheochromocytoma (PHEO)

$S D$ - standard deviation; $\mathrm{Cl}$ - confidence interval.

$p=0.80)$. A meta-analysis of these studies showed shorter length of hospital stay for RA (WMD $=-0.51$ days, $95 \% \mathrm{Cl}:-0.91$ to $-0.12 ; p=0.01$ ) (Figure 5 ).

There was no significant publication bias in this study.

\section{Discussion}

Adrenalectomy is still the gold standard treatment for PHEO. There are several different techniques available for adrenalectomy: open adrenalectomy, laparoscopic adrenalectomy, and robotic adrenalectomy. Laparoscopic adrenalectomy has been widely used and considered the gold standard for adrenal tumours [5, 14]. However, RA has the advantages of wristed instrument, freedom of movement, amplification effect, and highly visual three-dimensional vision $[6,15]$. Whether $R A$ is feasible to LA remains controversial. Therefore, we conducted a meta-analysis of the existing literature and drew objective conclusions on this topic.
In this meta-analysis, patients in the RA group and the LA group were similar in terms of age, gender, BMI, and tumour side. The tumour size was larger in the RA group. This could be the inherent selection bias due to the operative advantages of RA. Most of the studies included in this article reported large PHEOs. The mean tumour size was larger than $4 \mathrm{~cm}$ or even $8 \mathrm{~cm}$. Large PHEOs might be related to intense vascularization, adhesion with surrounding tissues, and contracting with inferior vena cava [16]. These would significantly increase the difficulty of dissection. However, we found similar operative time, EBL, transfusion rate, and conversion rate between the 2 groups. This could reflect the superiority of RA in dissection.

Most of the PHEOs are rich in blood vessels and catecholamine-secreting tumours. Haemodynamic crisis is the most challenging factor during the manipulation of the tumour $[17,18]$. Due to the lack of 


\section{Complications}

\begin{tabular}{|c|c|c|c|c|c|c|}
\hline \multirow{2}{*}{$\begin{array}{l}\text { Study } \\
\text { or subgroup }\end{array}$} & \multicolumn{2}{|c|}{ RA } & \multicolumn{2}{|c|}{ LA } & \multirow{2}{*}{$\begin{array}{c}\text { Weight } \\
\text { (\%) }\end{array}$} & \multirow{2}{*}{$\begin{array}{c}\text { Odds ratio } \\
\mathrm{M}-\mathrm{H} \text {, fixed, } 95 \% \mathrm{Cl}\end{array}$} \\
\hline & Events & Total & Events & Total & & \\
\hline Aliyev 2013 & 0 & 25 & 4 & 40 & 13.5 & $0.16(0.01,3.09)$ \\
\hline Fang 2020 & 11 & 41 & 23 & 89 & 41.8 & $1.05(0.46,2.43)$ \\
\hline Fu 2020 & 6 & 19 & 9 & 32 & 18.1 & $1.18(0.34,4.06)$ \\
\hline Ma 2020 & 11 & 70 & 8 & 70 & 26.6 & $1.44(0.54,3.84)$ \\
\hline Total $(95 \% \mathrm{Cl})$ & & 155 & & 231 & 100.0 & $1.06(0.62,1.82)$ \\
\hline Total events & 28 & & 44 & & & \\
\hline $\begin{array}{l}\text { Heterogeneity: } \\
\text { Test for overall }\end{array}$ & $\begin{array}{l}=1.99 \\
\text { ect: } Z\end{array}$ & $=$ & $\begin{array}{c}=0.57) \\
0.84)\end{array}$ & $1^{2}=0$ & & \\
\hline
\end{tabular}

\section{Clavien Dindo score $\geq 3$ complications}

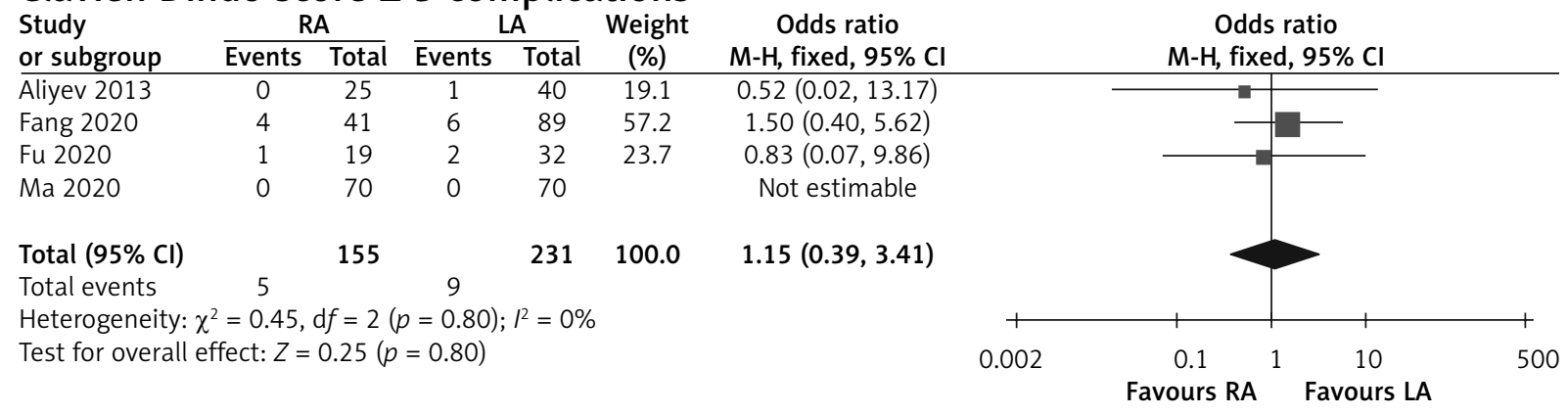

\section{Length of hospital stay (days)}

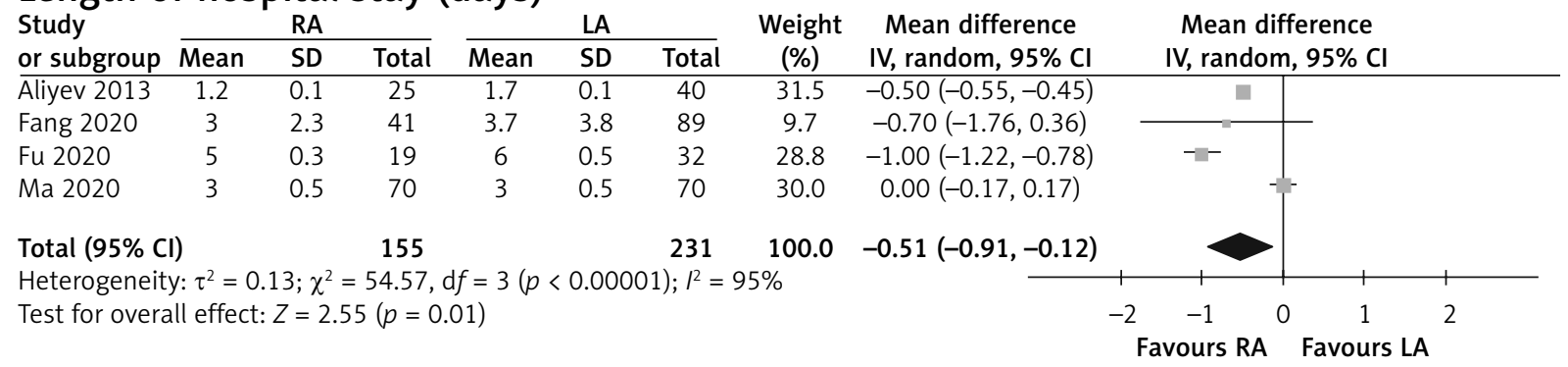

Figure 5. Forest and funnel plots of post-operative outcomes of robotic adrenalectomy (RA) vs. laparoscopic adrenalectomy (LA) for pheochromocytoma (PHEO)

tactile feedback, many researchers were concerned about the risk of bleeding, cardiovascular accidents, and intraoperative hypertensive crisis during the dissection of PHEO [8]. Our study found similar peri-operative haemodynamic outcomes between the 2 groups. This could be explained by the following factors. First, the patients were well prepared for surgery with $\alpha$-blockers (such as phenoxybenzamine), which could significantly improve perioperative haemodynamic fluctuations [19]. Second, with the help of a wristed instrument and three-dimensional vision, direct tumour manipulation was reduced. Therefore, after adequate preparation, both RA and LA could obtain good perioperative haemodynamic results. These results proved the safety of RA compared with LA.

Our study found that RA did not reduce the complications and Clavien-Dindo score $\geq 3$ complications. However, the length of hospital stay in the RA group was significantly shorter than that in the LA group. This could be related to the precise dissection and less interference of RA. This demonstrated that the efficacy of RA was similar to that of LA.

This study has several limitations. First, most of the studies were retrospective, which limited the quality of the results. Second, the small sample size of patients reduced the reliability of the results. Third, preoperative fluid and hypotensive drug ad- 
ministration were not standardized. Fourth, the robotic or laparoscopic approaches were not consistent. Fifth, it was not possible to analyse the oncological results. Sixth, long follow-up was required to verify the results. And last but not least, we did not have sufficient data to analyse the learning curve and cost. More multicentre randomized controlled trials are needed to focus on this topic, especially haemodynamic stability.

\section{Conclusions}

RA is as safe and feasible as LA regarding efficacy and safety for PHEO. In addition, RA might have advantages in handling large PHEOs. Nevertheless, more prospective randomized comparative studies are needed to investigate the role of RA for PHEO.

\section{Acknowledgments}

We thank Dr. Chawnshang Chang at University of Rochester Medical Centre for helping with the preparation of the manuscript.

\section{Conflict of interest}

The authors declare no conflict of interest.

\section{References}

1. Lenders JW, Eisenhofer G, Mannelli M, et al. Phaeochromocyto ma. Lancet 2005; 366: 665-75.

2. Farrugia FA, Martikos G, Tzanetis P, et al. Pheochromocytoma diagnosis and treatment: review of the literature. Endocr Regul 2017; 51: 168-81.

3. Schurmeyer TH, Engeroff B, Dralle H, et al. Cardiological effects of catecholamine-secreting tumours. Eur J Clin Invest 1997; 27: 189-95.

4. Zeiger MA, Thompson GB, Duh QY, et al. American Association of Clinical Endocrinologists and American Association of Endocrine Surgeons Medical Guidelines for the Management of adrenal Incidentalomas: executive summary of recommendations. Endocr Pract 2009; 15: 450-3.

5. Li J, Wang Y, Chang X, et al. Laparoscopic adrenalectomy (LA) vs open adrenalectomy (OA) for pheochromocytoma (PHEO): a systematic review and meta-analysis. Eur J Surg Oncol 2020; 46: 991-8.

6. Woo Y, Hyung WJ, Pak KH, et al. Robotic gastrectomy as an oncologically sound alternative to laparoscopic resections for the treatment of early-stage gastric cancers. Arch Surg 2011; 146: 1086-92.

7. Morris LF, Perrier ND. Advances in robotic adrenalectomy. Curr Opin Oncol 2012; 24: 1-6.
8. Bihain F, Klein M, Nomine-Criqui C, et al. Robotic adrenalectomy in patients with pheochromocytoma: a systematic review. Gland Surg 2020; 9: 844-8.

9. Hozo SP, Djulbegovic B, Hozo I. Estimating the mean and variance from the median, range, and the size of a sample. BMC Med Res Methodol 2005; 5: 13.

10. Aliyev S, Karabulut K, Agcaoglu O, et al. Robotic versus laparoscopic adrenalectomy for pheochromocytoma. Ann Surg Oncol 2013; 20: 4190-4.

11. Fang AM, Rosen J, Saidian A, et al. Perioperative outcomes of laparoscopic, robotic, and open approaches to pheochromocytoma. Robot Surg 2020; 14: 849-54.

12. Fu SQ, Zhuang CS, Yang XR, et al. Comparison of robot-assisted retroperitoneal laparoscopic adrenalectomy versus retroperitoneal laparoscopic adrenalectomy for large pheochromocytoma: a single-centre retrospective study. BMC Surg 2020; 20: 227.

13. Ma W, Mao Y, Zhuo R, et al. Surgical outcomes of a randomized controlled trial compared robotic versus laparoscopic adrenalectomy for pheochromocytoma. Eur J Surg Oncol 2020; 46: 1843-7.

14. Gagner M, Lacroix A, Bolte E. Laparoscopic adrenalectomy in Cushing's syndrome and pheochromocytoma. N Engl J Med 1992; 327: 1033.

15. Yu HY, Friedlander DF, Patel S, et al. The current status of robotic oncologic surgery. CA Cancer J Clin 2013; 63: 45-56.

16. Taffurelli G, Ricci C, Casadei R, et al. Open adrenalectomy in the era of laparoscopic surgery: a review. Updates Surg 2017; 69: 135-43.

17. Sun F, Zhuo R, Ma W, et al. From clinic to mechanism: proteomics-based assessment of angiogenesis in adrenal pheochromocytoma. J Cell Physiol 2019; 234: 22057-70.

18. Chai YJ, Yu HW, Song RY, et al. Lateral transperitoneal adrenalectomy versus posterior retroperitoneoscopic adrenalectomy for benign adrenal gland disease: randomized controlled trial at a single tertiary medical centre. Ann Surg 2019; 269: 842-8.

19. Pacak K. Preoperative management of the pheochromocytoma patient. J Clin Endocrinol Metab 2007; 92: 4069-79.

Received: 21.05.2021, accepted: 15.06.2021. 